\title{
Metallic antimony (Sb) by XPS
}

David J. Morgan

Citation: Surface Science Spectra 24, 024004 (2017);

View online: https://doi.org/10.1116/1.4994636

View Table of Contents: http://avs.scitation.org/toc/sss/24/2

Published by the American Vacuum Society

\section{Articles you may be interested in}

Cluster cleaned HOPG by XPS

Surface Science Spectra 24, 024003 (2017); 10.1116/1.4993771

Introduction to a series of dicarboxylic acids analyzed by $x$-ray photoelectron spectroscopy

Surface Science Spectra 24, 011001 (2017); 10.1116/1.4983448

Vanadium, niobium and tantalum by XPS

Surface Science Spectra 24, 024001 (2017); 10.1116/1.4998018

\section{Instruments for Advanced Science}

Contact Hiden Analytical for further details: w www.HidenAnalytical.com E info@hiden.co.uk CLICK TO VIEW our product catalogue

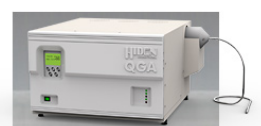

Gas Analysis

dynamic measurement of reaction gas streams catalysis and thermal analysis

molecular beam studies

dissolved species probes

, dermentation

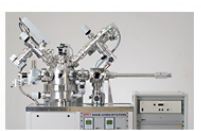

Surface Science

UHVTPD

SIMS elemental imaging - surface mapping

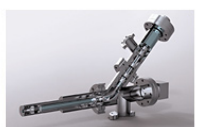

Plasma Diagnostics

plasma source characterization etch and deposition process reaction , kinetic studies

analysis of neutral and radical species

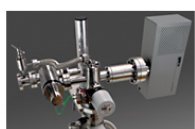

Vacuum Analysis partial pressure measurement and contro of process gases , reactive sputter process control , vacuum diagnostics , vacuum coating process monitoring 


\section{Metallic antimony (Sb) by XPS}

David J. Morgan ${ }^{\text {a) }}$

Cardiff Catalysis Institute, School of Chemistry, Cardiff University, Park Place, Cardiff CF10 3AT, United Kingdom

(Received 6 July 2017; accepted 13 September 2017; published 17 October 2017)

Herein, survey and high-resolution core level, x-ray induced Auger, and valence photoelectron spectra recorded from a section of a mechanically polished antimony ( $\mathrm{Sb}$ ) rod are presented using monochromatic Al radiation. (C) 2017 American Vacuum Society.

https://doi.org/10.1116/1.4994636

Keywords: Antimony, XPS, metal
Accession \#: 1422
Technique: XPS
Host Material: Antimony (Sb)
Instrument: Thermo Scientific K-Alpha+
Major Elements in Spectra: $\mathrm{Sb}$
Minor Elements in Spectra: C
Published Spectra: 10
Spectra in Electronic Record: 10
Spectral Category: Reference

\section{INTRODUCTION}

Metallic antimony ( $\mathrm{Sb}$ ) is typically widely used in $\mathrm{Sn}-\mathrm{Pb}$ alloys found in lead-acid batteries and used to quench hydrogen production and improve the characteristics of charging (Ref. 1). Further uses include alloys for antifriction applications (e.g., bullets or bearings from Babbitt-type metal alloys) (Ref. 2) and lead-free solders (Ref. 3) and as a fining agent for specialist glass production (Ref. 4). The toxicity of antimony is well documented (Ref. 5), which has potentially limited surface analysis to typically inorganic antimony materials (Ref. 6) and Sb containing semiconductors/photoconductors (Ref. 7).

The presented spectra serve as an up to date reference for oxygen free metallic $\mathrm{Sb}$, which hitherto has been typically overlooked for analysis with the latest generation of photoelectron spectrometers.

For both proof and clarity of the absence of oxygen, Fig. 1 shows closeup sections of survey spectra from the presented data and Sb-containing oxides, which, due to weak Auger features close to the O KLL region, may be mistaken for oxygen.

\section{SPECIMEN DESCRIPTION (ACCESSION \# 1422)}

Host Material: Antimony (Sb)

CAS Registry \#: 7440-36-0

Host Material Characteristics: Homogeneous; solid; polycrystalline; conductor; metal; others

Chemical Name: Antimony

Source: Unknown

Host Composition: $\mathrm{Sb}$

Form: Rod

\section{Structure: $\mathrm{Sb}$}

History and Significance: The rod was one of the many stored in a screw-top glass jar for an unknown period of time. Spectra were recorded as the basis of reference spectra for oxidation and valence band studies.

As Received Condition: The rod was approximately $50 \mathrm{~mm}$ in length and $5 \mathrm{~mm}$ in diameter. The appearance of the rod was a dull gray color, indicating surface oxidation.

Analyzed Region: Center of the host material

a)Electronic mail: MorganDJ3@ @ cardiff.ac.uk

\section{Ex Situ Preparation/Mounting:}

The sample was cleaved using a sharp blade after immersion of the rod in liquid nitrogen. The cleaved area was a lustrous silver color displaying a highly faceted area. The cleaved sample was wet polished using SiC paper until a visibly flat surface was obtained. The sample was subsequently rinsed and further ultrasonically cleaned with iso-propyl alcohol. The cleaned sample was mounted onto a sample plate using double sided conductive carbon tape.

In Situ Preparation: $4 \mathrm{kV}$ argon ion sputtering over an area of 4 $\times 4 \mathrm{~mm}$. Cleanliness was assumed once no O KLL signal was detected in the survey spectra or notable contribution of antimony oxide to the $\mathrm{Sb} 3 \mathrm{~d}$ spectrum.

Charge Control: None required as conducting

Temp. During Analysis: $300 \mathrm{~K}$

Pressure During Analysis: $5.1 \times 10^{-7} \mathrm{~Pa}$

Preanalysis Beam Exposure: The analyzed region was exposed to less than $60 \mathrm{~s}$ of beam exposure during the analysis of autoheight procedures.

\section{INSTRUMENT DESCRIPTION}

Manufacturer and Model: Thermo Scientific K-Alpha+

Analyzer Type: Double focussing hemispherical analyzer

Detector: Multichannel resistive plate

Number of Detector Elements: 128

INSTRUMENT PARAMETERS COMMON TO ALL SPECTRA -

\section{- Spectrometer}

Analyzer Mode: Constant pass energy

Throughput $\left(T=E^{\mathrm{N}}\right)$ : Other

Excitation Source Window: None

Excitation Source: Al Ka monochromatic

Source Energy: $1486.6 \mathrm{eV}$

Source Strength: $72 \mathrm{~W}$

Source Beam Size: $400 \times 400 \mu \mathrm{m}$

Signal Mode: Multichannel direct

Geometry

Incident Angle: $60^{\circ}$

Source-to-Analyzer Angle: $60^{\circ}$ 


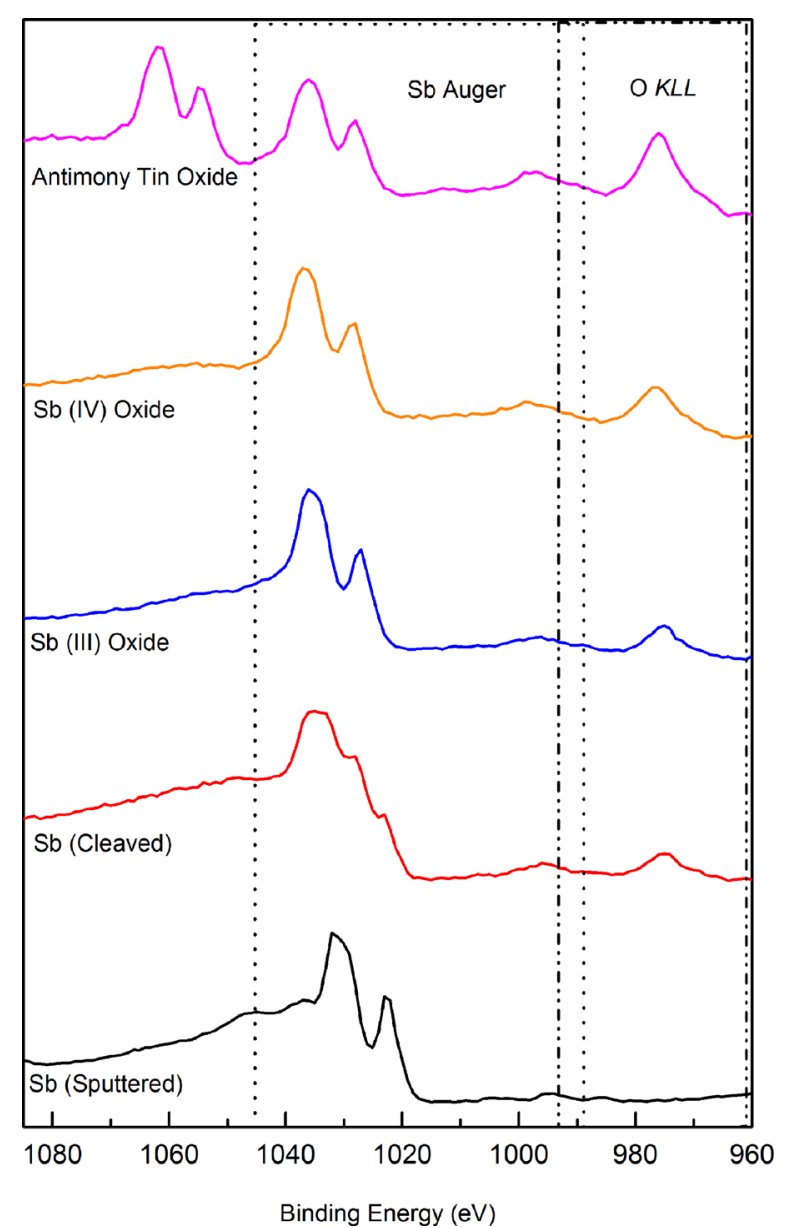

FIG. 1. (Color online) Sections of survey spectra for metallic Sb and associated $\mathrm{Sb}$ containing oxides.

Emission Angle: $90^{\circ}$

Specimen Azimuthal Angle: $45^{\circ}$

Acceptance Angle from Analyzer Axis: $60^{\circ}$

Analyzer Angular Acceptance Width: $45^{\circ} \times 0^{\circ}$

\section{Ion gun}

Manufacturer and Model: Thermo Scientific MAGCIS

Energy: $4000 \mathrm{eV}$

Current: $4.2 \mathrm{~mA}$
Current Measurement Method: Faraday cup

Sputtering Species: $\mathrm{Ar}^{+}$

Spot Size (unrastered): $120 \mu \mathrm{m}$

Raster Size: $4000 \times 4000 \mu \mathrm{m}$

Incident Angle: $58^{\circ}$

Polar Angle: $58^{\circ}$

Azimuthal Angle: $90^{\circ}$

Comment: Enter ion beam comment.

DATA ANALYSIS METHOD

Energy Scale Correction: Not required

Recommended Energy Scale Shift: Not required

Peak Shape and Background Method: CasaXPS (v2.3.19 rev1.1h) was used to perform background subtraction using a Shirley background and the determination of peak positions and the full width at half maximum (FWHM) values. Asymmetric peaks were used to fit the Sb $3 \mathrm{~d}$ core levels, while Gaussian peaks were used for all other levels.

Quantitation Method: Atomic concentrations were calculated in CasaXPS using Scofield sensitivity factors with an energy dependence of -0.6 .

\section{ACKNOWLEDGMENTS}

The author gratefully acknowledges the Ser Cymru Capital Equipment Fund for help in the purchase of the spectrometer.

\section{REFERENCES}

1. A. H. Kiehne, Battery Technology Handbook (CRC, USA, 2003).

2. R. S. Williams, Principles of Metallography (Read Books, United Kingdom, 2007).

3. H. Ipser, H. Flandorfer, Ch. Luef, C. Schetterer, and U. Saeed, J. Mater. Sci.: Mater. Electron. 18, 3 (2007).

4. H. Yamashita, S. Yamaguchi, R. Nishimura, and T. Maekawa, Anal. Sci. 17, 45 (2001).

5. S. Sundar and J. Chakravaty, Int. J. Environ. Res. Public Health 7, 4267 (2010).

6. F. Garbassi, Surf. Interface Anal. 2, 165 (1980).

7. R. G. Copperthwaite, O. A. Junze, J. Lloyd, J. A. Neely, and W. Tuma, Z. Naturf. A 33, 523 (1978). 


\begin{tabular}{|c|c|c|c|c|c|c|c|}
\hline \multicolumn{8}{|c|}{ SPECTRAL FEATURE TABLE } \\
\hline $\begin{array}{l}\text { Spectrum } \\
\text { ID \# }\end{array}$ & $\begin{array}{l}\text { Element/ } \\
\text { Transition }\end{array}$ & $\begin{array}{l}\text { Peak } \\
\text { Energy } \\
(\mathrm{eV})\end{array}$ & $\begin{array}{c}\text { Peak } \\
\text { Width } \\
\text { FWHM } \\
(\mathrm{eV})\end{array}$ & $\begin{array}{c}\text { Peak } \\
\text { Area } \\
(\mathrm{eV} \text { cts } / \mathbf{s})\end{array}$ & $\begin{array}{l}\text { Sensitivity } \\
\text { Factor }\end{array}$ & $\begin{array}{c}\text { Concentration } \\
\text { (at. \%) }\end{array}$ & $\begin{array}{c}\text { Peak } \\
\text { Assignment }\end{array}$ \\
\hline N1422_01 & $\mathrm{Sb} 4 \mathrm{~d}$ & 33.1 & $\cdots$ & $\cdots$ & $\cdots$ & $\cdots$ & $\cdots$ \\
\hline N1422_01 & $\mathrm{Sb} 4 \mathrm{p}$ & 100.2 & $\cdots$ & $\cdots$ & $\cdots$ & $\cdots$ & $\cdots$ \\
\hline N1422_01 & $\mathrm{Sb} 4 \mathrm{~s}$ & 153.1 & $\ldots$ & $\ldots$ & $\ldots$ & $\ldots$ & $\ldots$ \\
\hline N1422_01 & $\mathrm{Sb} 3 \mathrm{~d}_{5 / 2}$ & 528.1 & $\cdots$ & $\cdots$ & $\cdots$ & $\cdots$ & $\cdots$ \\
\hline N1422_01 & $\mathrm{Sb} 3 \mathrm{~d}_{3 / 2}$ & 538.1 & $\ldots$ & $\ldots$ & $\ldots$ & $\ldots$ & $\ldots$ \\
\hline N1422_01 & $\mathrm{Sb} 3 \mathrm{p}_{3 / 2}$ & 766.2 & $\cdots$ & $\cdots$ & $\cdots$ & $\cdots$ & $\cdots$ \\
\hline N1422_01 & $\mathrm{Sb} 3 p_{1 / 2}$ & 812.6 & $\ldots$ & $\ldots$ & $\ldots$ & $\ldots$ & $\ldots$ \\
\hline N1422_01 & $\mathrm{Sb} 3 \mathrm{~s}$ & 945.5 & $\cdots$ & $\cdots$ & $\cdots$ & $\ldots$ & $\ldots$ \\
\hline N1422_01 & Sb Auger & 985 & $\cdots$ & $\ldots$ & $\ldots$ & $\ldots$ & $\ldots$ \\
\hline N1422_01 & Sb Auger & 1022.1 & $\ldots$ & $\ldots$ & $\ldots$ & $\ldots$ & $\ldots$ \\
\hline N1422_01 & Sb Auger & 1031.2 & $\cdots$ & $\cdots$ & $\ldots$ & $\cdots$ & $\cdots$ \\
\hline N1422_02 & Valence & $\cdots$ & $\cdots$ & $\cdots$ & $\cdots$ & $\cdots$ & Valence band \\
\hline N1422_03 & $\mathrm{Sb} 4 \mathrm{~d}_{5 / 2}$ & 32.0 & 0.87 & 391845.10 & 1.85 & $\ldots$ & Sb metal \\
\hline N1422_03 & $\mathrm{Sb} 4 \mathrm{~d}_{3 / 2}$ & 33.3 & 0.87 & 261243.10 & 1.29 & $\ldots$ & Sb metal \\
\hline N1422_04 ${ }^{a}$ & $\mathrm{Sb} 4 p$ & 99.0 & $\ldots$ & $\ldots$ & 2.88 & $\ldots$ & Sb metal \\
\hline N1422_05 & $\mathrm{Sb} 4 \mathrm{~s}$ & 152.8 & 3.61 & 55128.60 & 0.85 & $\ldots$ & Sb metal \\
\hline N1422_06 & C 1s & 284.5 & 1.75 & 4451.7 & 1.00 & $\ldots$ & $\begin{array}{l}\text { Residual carbon, } \\
\text { possibly implanted } \\
\text { by the ion beam }\end{array}$ \\
\hline N1422_07 & $\mathrm{Sb} 3 \mathrm{~d}_{5 / 2}$ & 528.1 & 0.77 & 29822576.9 & 16.39 & $\ldots$ & Sb metal \\
\hline N1422_07 & $\mathrm{Sb} 3 \mathrm{~d}_{3 / 2}$ & 537.4 & 0.76 & 1988384.6 & 11.35 & $\ldots$ & Sb metal \\
\hline N1422_07 ${ }^{\mathrm{b}}$ & $\mathrm{Sb} 3 d_{5 / 2}$ satellite & 543.8 & 5.07 & 691459.0 & $\ldots$ & $\ldots$ & Sb $3 d_{5 / 2}$ satellite \\
\hline N1422_07 ${ }^{b}$ & $\mathrm{Sb} 3 \mathrm{~d}_{3 / 2}$ satellite & 553.2 & 1.71 & 58845.2 & $\ldots$ & $\ldots$ & $\mathrm{Sb} 3 \mathrm{~d}_{3 / 2}$ satellite \\
\hline N1422_08 ${ }^{\mathrm{b}}$ & $\mathrm{Sb} 3 p_{3 / 2}$ & 766.0 & 3.33 & 1589019.4 & 9.77 & $\ldots$ & Sb metal \\
\hline N1422_08 & $\mathrm{Sb} 3 p_{3 / 2}$ Loss & 782.0 & 8.39 & 514954.3 & $\ldots$ & $\ldots$ & $\mathrm{Sb} 3 \mathrm{p}_{3 / 2}$ loss peak (1) \\
\hline N1422_08 ${ }^{\mathrm{b}}$ & $\mathrm{Sb} 3 p_{3 / 2}$ Loss & 797.9 & 4.15 & 23715.3 & $\ldots$ & $\ldots$ & $\mathrm{Sb} 3 \mathrm{p}_{3 / 2}$ loss peak (2) \\
\hline N1422_08 ${ }^{\mathrm{b}}$ & $\mathrm{Sb} 3 \mathrm{p}_{1 / 2}$ & 812.2 & 3.33 & 794509.7 & 4.76 & $\ldots$ & Sb metal \\
\hline N1422_08 ${ }^{\mathrm{b}}$ & $\mathrm{Sb} 3 p_{1 / 2}$ Loss & 828.2 & 8.39 & 257477.1 & $\ldots$ & $\ldots$ & $\mathrm{Sb} 3 \mathrm{p}_{3 / 2}$ loss peak (1) \\
\hline N1422_08 ${ }^{\mathrm{b}}$ & $\mathrm{Sb} 3 p_{1 / 2}$ Loss & 844.1 & 4.15 & 11857.6 & $\ldots$ & $\ldots$ & $\mathrm{Sb} 3 \mathrm{p}_{3 / 2}$ loss peak (2) \\
\hline N1422_09 ${ }^{c}$ & $\mathrm{Sb} 3 \mathrm{~s}$ & 945.1 & $\ldots$ & $\ldots$ & $\ldots$ & $\ldots$ & $\mathrm{Sb}$ metal \\
\hline N1422_10 & Sb MNN & 1022.4 & $\cdots$ & $\ldots$ & $\ldots$ & $\ldots$ & $\mathrm{Sb} \mathrm{M} \mathrm{N}_{45} \mathrm{~N}_{45}$ \\
\hline N1422_10 ${ }^{d}$ & Sb MNN & 1031.7 & $\ldots$ & $\ldots$ & $\ldots$ & $\ldots$ & $\mathrm{Sn} \mathrm{M}_{5} \mathrm{~N}_{45} \mathrm{~N}_{45}$ \\
\hline
\end{tabular}

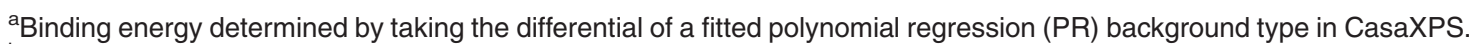

${ }^{\mathrm{b}}$ For quantification purposes, the satellite structure should be included in the peak area calculation if the metallic content is sufficiently high.

${ }^{c}$ Very broad structured peak, binding energy taken to be the highest peak position.

${ }^{\mathrm{d}} \mathrm{Sb}$ also exhibits weak Auger transitions around $945 \mathrm{eV}$, which are not to be confused with neighboring O KLL Auger peaks. 


\begin{tabular}{lccccccc}
\hline \multicolumn{7}{c}{ ANALYZER CALIBRATION TABLE } \\
\hline $\begin{array}{l}\text { Spectrum } \\
\text { ID \# }\end{array}$ & $\begin{array}{c}\text { Element/ } \\
\text { Transition }\end{array}$ & $\begin{array}{c}\text { Peak Energy } \\
(\mathbf{e V})\end{array}$ & $\begin{array}{c}\text { Peak } \\
\text { Width } \\
\text { FWHM (eV) }\end{array}$ & $\begin{array}{c}\text { Peak Area } \\
\text { (eV cts/s) }\end{array}$ & $\begin{array}{c}\text { Sensitivity } \\
\text { Factor }\end{array}$ & $\begin{array}{c}\text { Concentration } \\
\text { (at. \%) }\end{array}$ & $\begin{array}{c}\text { Peak } \\
\text { Assignment }\end{array}$ \\
\hline 1 & $\mathrm{Au} \mathrm{4f_{7/2 }}$ & 83.99 & 0.76 & 1597652 & 9.58 & 100 & Gold metal \\
2 & $\mathrm{Ag} \mathrm{3d_{5/2 }}$ & 368.28 & 0.58 & 1876744 & 7.38 & 100 & Silver metal \\
3 & $\mathrm{Cu} 2 \mathrm{p}_{3 / 2}$ & 932.67 & 0.83 & 2205571 & 16.73 & 100 & Copper metal \\
\hline
\end{tabular}

\begin{tabular}{lccccc}
\hline \multicolumn{5}{c}{ GUIDE TO FIGURES } \\
\hline Spectrum (Accession) \# & Spectral Region & Voltage Shift & Multiplier & Baseline & Comment \# \\
\hline N1422_01 & Survey & 0 & 1 & 0 & $\ldots$ \\
N1422_02 & Valence & 0 & 1 & 0 & $\ldots$ \\
N1422_03 & Sb 4d & 0 & 1 & 0 & $\ldots$ \\
N1422_04 & Sb 4p & 0 & 1 & 0 & $\ldots$ \\
N1422_05 & Sb 4s & 0 & 1 & 0 & $\ldots$ \\
N1422_06 & C 1s & 0 & 1 & 0 & $\ldots$ \\
N1422_07 & Sb 3d & 0 & 1 & 0 & $\ldots$ \\
N1422_08 & Sb 3p & 0 & 1 & 0 & $\ldots$ \\
N1422_09 & Sb 3s & 0 & 1 & 0 & $\ldots$ \\
N1422_10 & Sb MNN & 0 & 1 & 0 & $\ldots$ \\
\hline
\end{tabular}




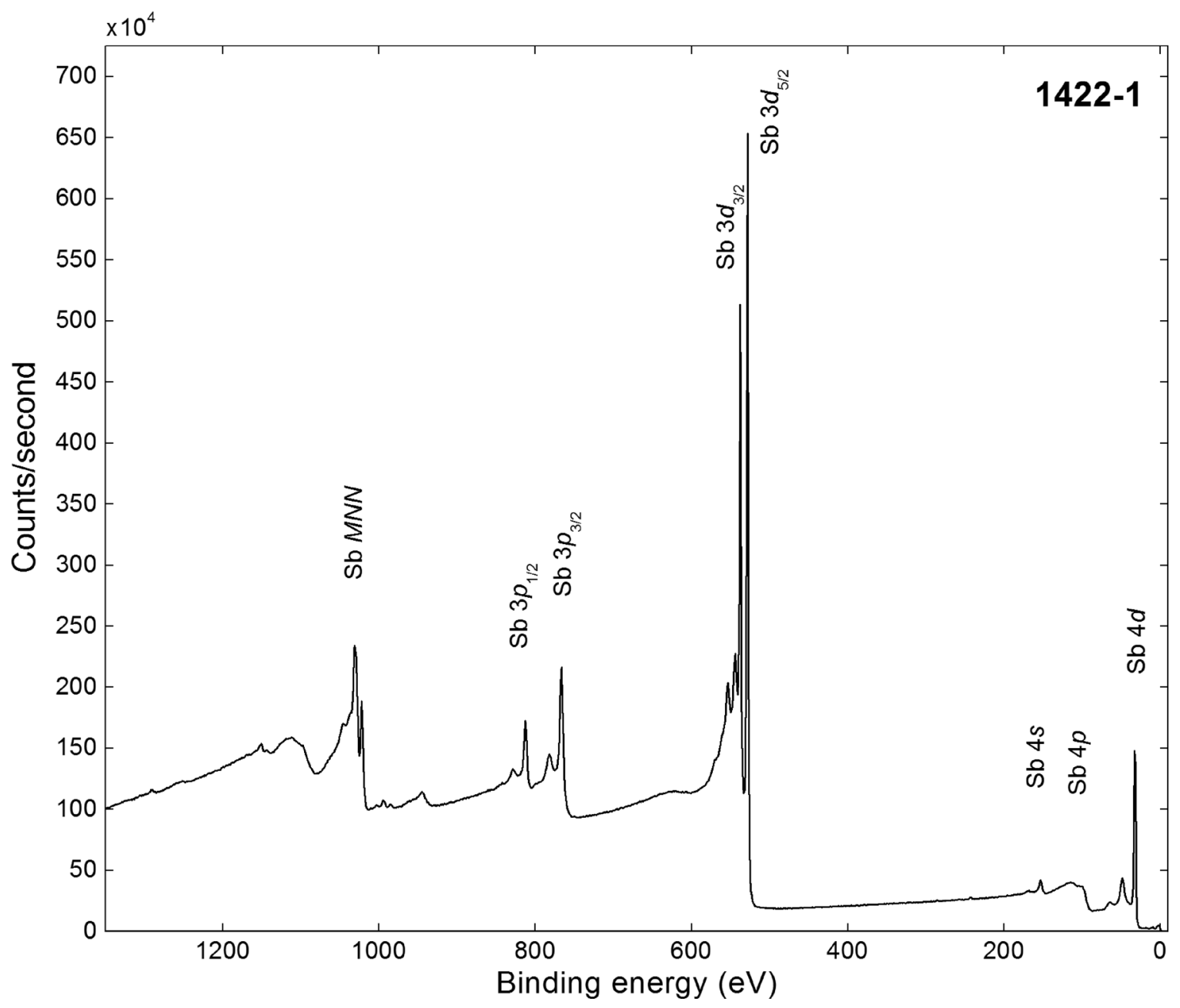

\begin{tabular}{rc}
\hline Accession \# & 1422-01 \\
Host Material & Antimony (Sb) \\
Technique & XPS \\
Spectral Region & Survey \\
Instrument & Thermo Scientific K-Alpha ${ }^{+}$ \\
Excitation Source & Al Ka monochromatic \\
Source Energy & $1486.6 \mathrm{eV}$ \\
Source Strength & $72 \mathrm{~W}$ \\
Source Size & $0.004 \times 0.004 \mathrm{~mm}$ \\
Analyzer Type & $60^{\circ}$ \\
Incident Angle & $90^{\circ}$ \\
Emission Angle & $150 \mathrm{eV}$ \\
Analyzer Pass Energy & $0.1 \mathrm{eV}$ \\
Analyzer Resolution & $130 \mathrm{~s}$ \\
Total Elapsed Time & $260 \mathrm{~s}$ \\
Number of Scans & 10 \\
Total Signal Accumulation Time & $1 \mathrm{eV}$ \\
\hline
\end{tabular}




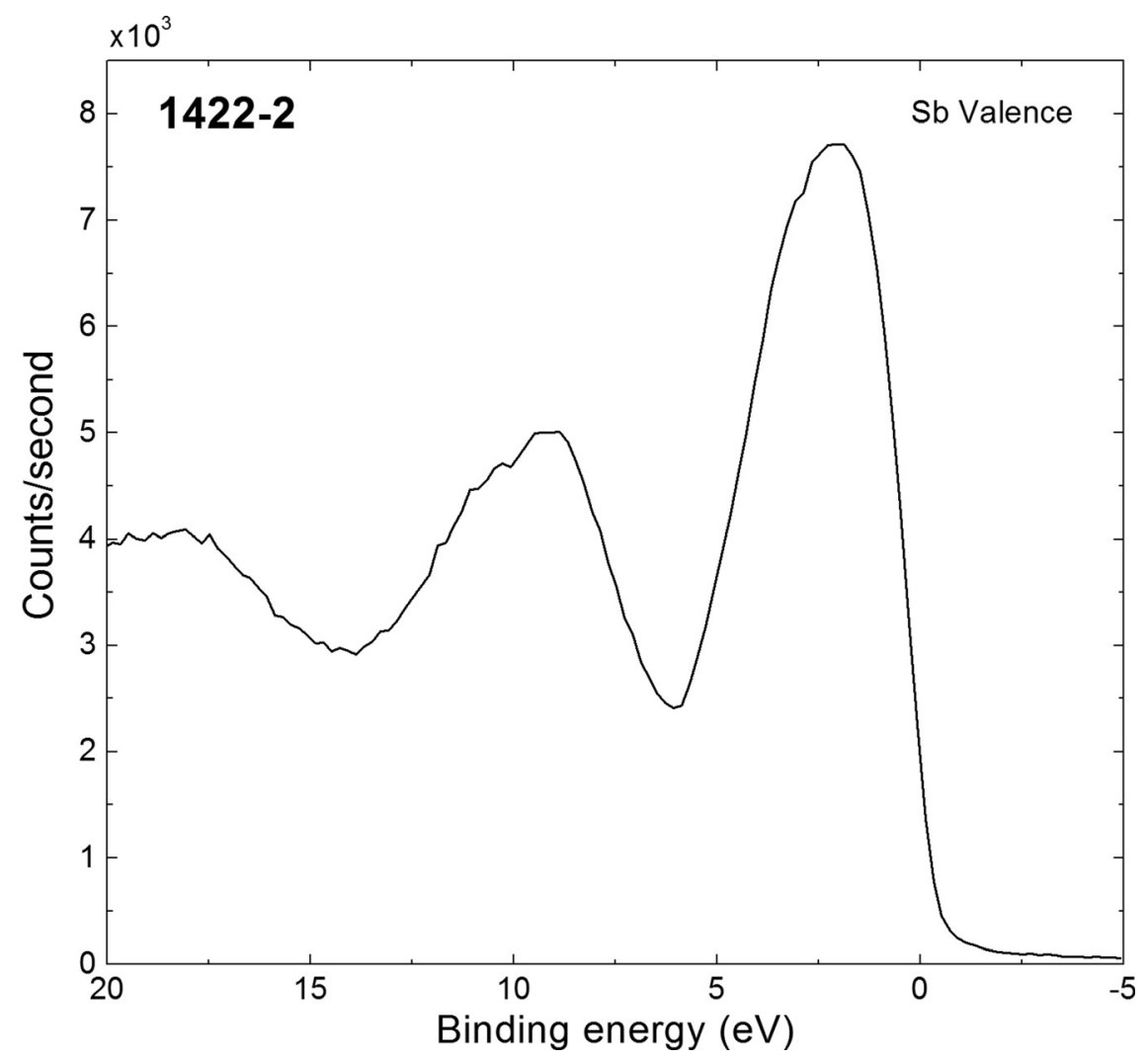

Accession \#: 1422-02

- Host Material: Antimony (Sb)

Technique: XPS

- Spectral Region: Valence

Instrument: Thermo Scientific K-Alpha+

Excitation Source: Al Ka monochromatic

Source Energy: $1486.6 \mathrm{eV}$

Source Strength: $72 \mathrm{~W}$

Source Size: $0.004 \times 0.004 \mathrm{~mm}$

Analyzer Type: Double focussing hemispherical analyzer

Incident Angle: $60^{\circ}$

Emission Angle: $90^{\circ}$

Analyzer Pass Energy: $40 \mathrm{eV}$

Analyzer Resolution: $0.1 \mathrm{eV}$

Total Signal Accumulation Time: $120 \mathrm{~s}$

Total Elapsed Time: $153 \mathrm{~s}$

Number of Scans: 20

Effective Detector Width: $0.1 \mathrm{eV}$

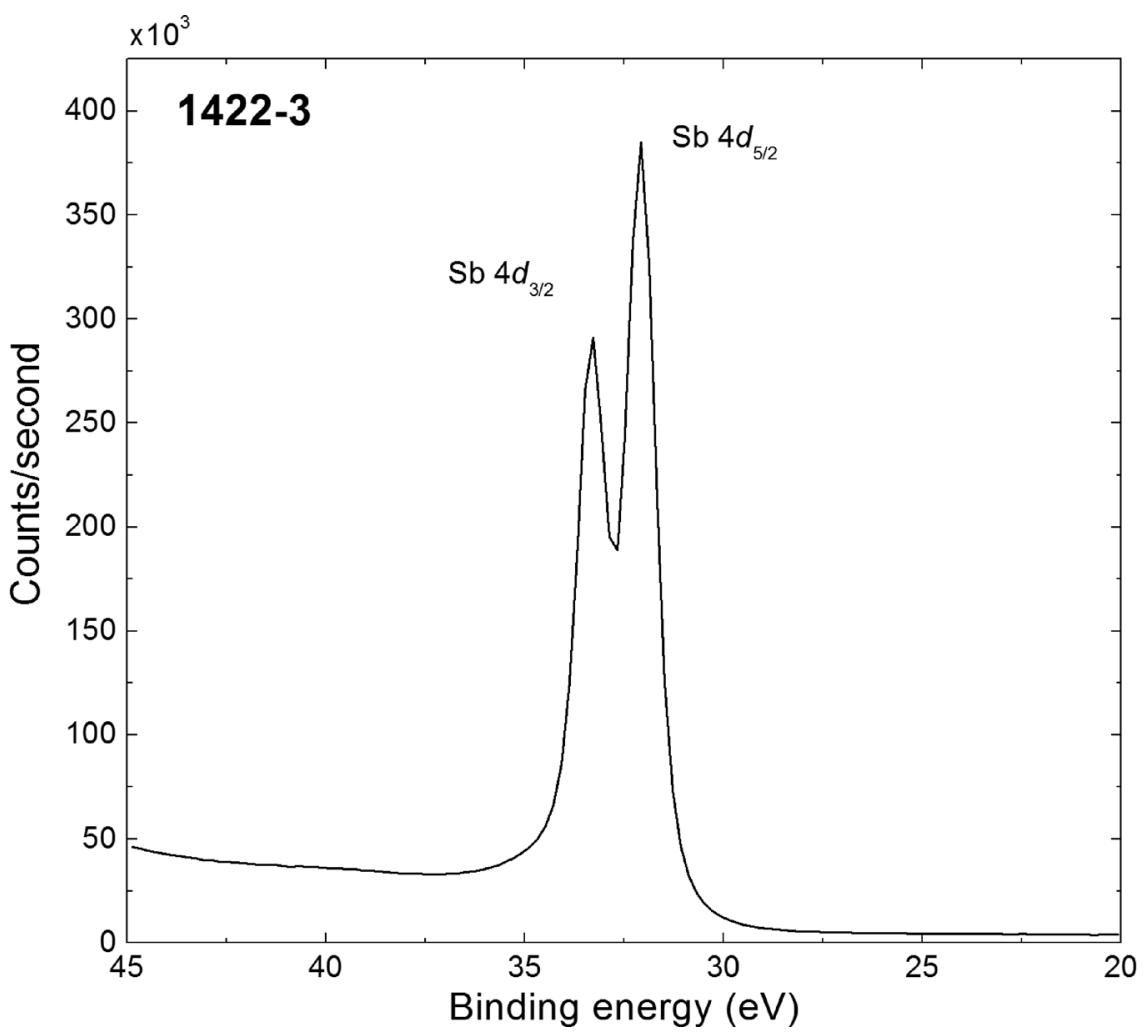

Accession \#: 1422-03

- Host Material: Antimony (Sb)

- Technique: XPS

- Spectral Region: Sb 4d

Instrument: Thermo Scientific K-Alpha+

Excitation Source: Al Ka monochromatic

Source Energy: $1486.6 \mathrm{eV}$

Source Strength: $72 \mathrm{~W}$

Source Size: $0.004 \times 0.004 \mathrm{~mm}$

Analyzer Type: Double focussing hemispherical analyzer

Incident Angle: $60^{\circ}$

Emission Angle: $90^{\circ}$

Analyzer Pass Energy: $40 \mathrm{eV}$

Analyzer Resolution: $0.1 \mathrm{eV}$

Total Signal Accumulation: Time: $120 \mathrm{~s}$

Total Elapsed Time: $153 \mathrm{~s}$

Number of Scans: 20

Effective Detector Width: $0.1 \mathrm{eV}$ 

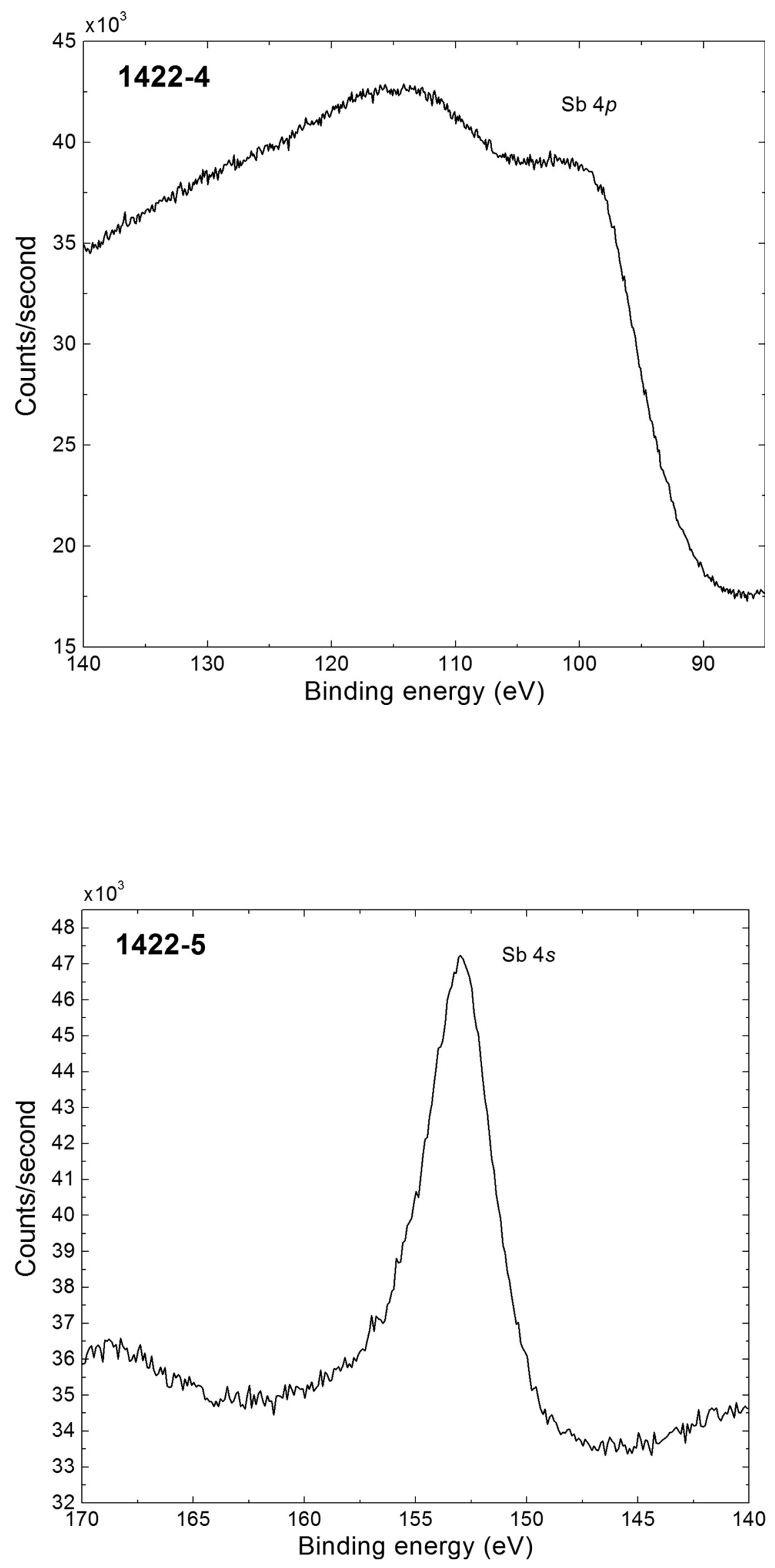

Accession \#: 1422-05

- Host Material: Antimony (Sb)

- Technique: XPS

- Spectral Region: Sb 4s

Instrument: Thermo Scientific K-Alpha+

Excitation Source: Al Ka monochromatic

Source Energy: $1486.6 \mathrm{eV}$

Source Strength: $72 \mathrm{~W}$

Source Size: $0.004 \times 0.004 \mathrm{~mm}$

Analyzer Type: Double focussing hemispherical analyzer

Incident Angle: $60^{\circ}$

Emission Angle: $90^{\circ}$

Analyzer Pass Energy: $40 \mathrm{eV}$

Analyzer Resolution: $0.1 \mathrm{eV}$

Total Signal Accumulation Time: $120 \mathrm{~s}$

Total Elapsed Time: $153 \mathrm{~s}$

Number of Scans: 20

Effective Detector Width: $0.1 \mathrm{eV}$ 


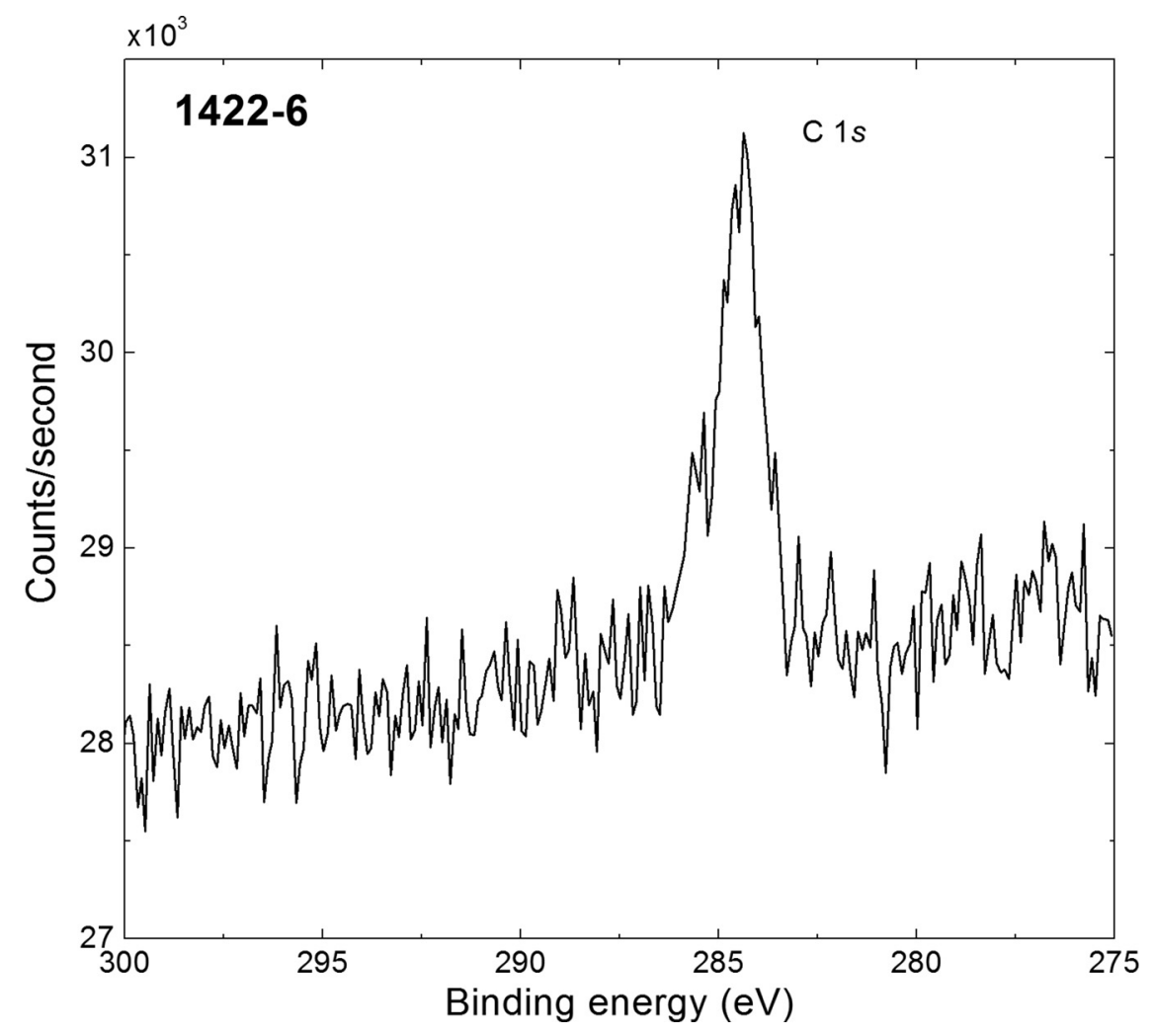

Accession \#: 1422-06

- Host Material: Antimony (Sb)

- Technique: XPS

- Spectral Region: C 1s

Instrument: Thermo Scientific

K-Alpha+

Excitation Source: Al Ka monochromatic

Source Energy: $1486.6 \mathrm{eV}$

Source Strength: $72 \mathrm{~W}$

Source Size: $0.004 \times 0.004 \mathrm{~mm}$

Analyzer Type: Double focussing

hemispherical analyzer

Incident Angle: $60^{\circ}$

Emission Angle: $90^{\circ}$

Analyzer Pass Energy: $40 \mathrm{eV}$

Analyzer Resolution: $0.1 \mathrm{eV}$

Total Signal Accumulation Time: $120 \mathrm{~s}$

Total Elapsed Time: $153 \mathrm{~s}$

Number of Scans: 20

Effective Detector Width: $0.1 \mathrm{eV}$

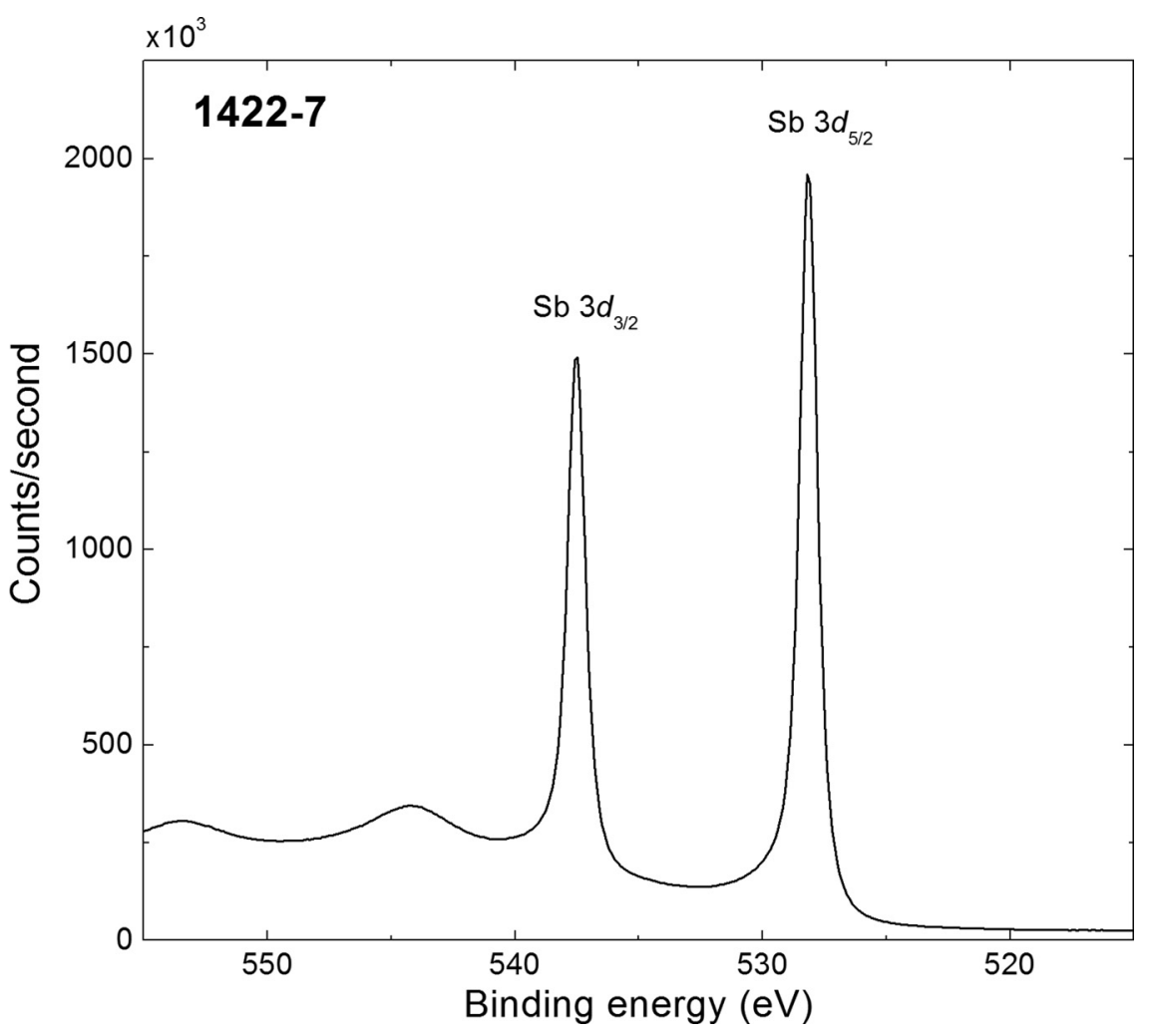

Accession \#: 1422-07

- Host Material: Antimony (Sb)

- Technique: XPS

- Spectral Region: Sb 3d

Instrument: Thermo Scientific K-Alpha+

Excitation Source: Al Ka monochromatic

Source Energy: $1486.6 \mathrm{eV}$

Source Strength: $72 \mathrm{~W}$

Source Size: $0.004 \times 0.004 \mathrm{~mm}$

Analyzer Type: Double focussing hemispherical analyzer

Incident Angle: $60^{\circ}$

Emission Angle: $90^{\circ}$

Analyzer Pass Energy: $40 \mathrm{eV}$

Analyzer Resolution: $0.1 \mathrm{eV}$

Total Signal Accumulation Time: $120 \mathrm{~s}$

Total Elapsed Time: $153 \mathrm{~s}$

Number of Scans: 20

Effective Detector Width: $0.1 \mathrm{eV}$ 


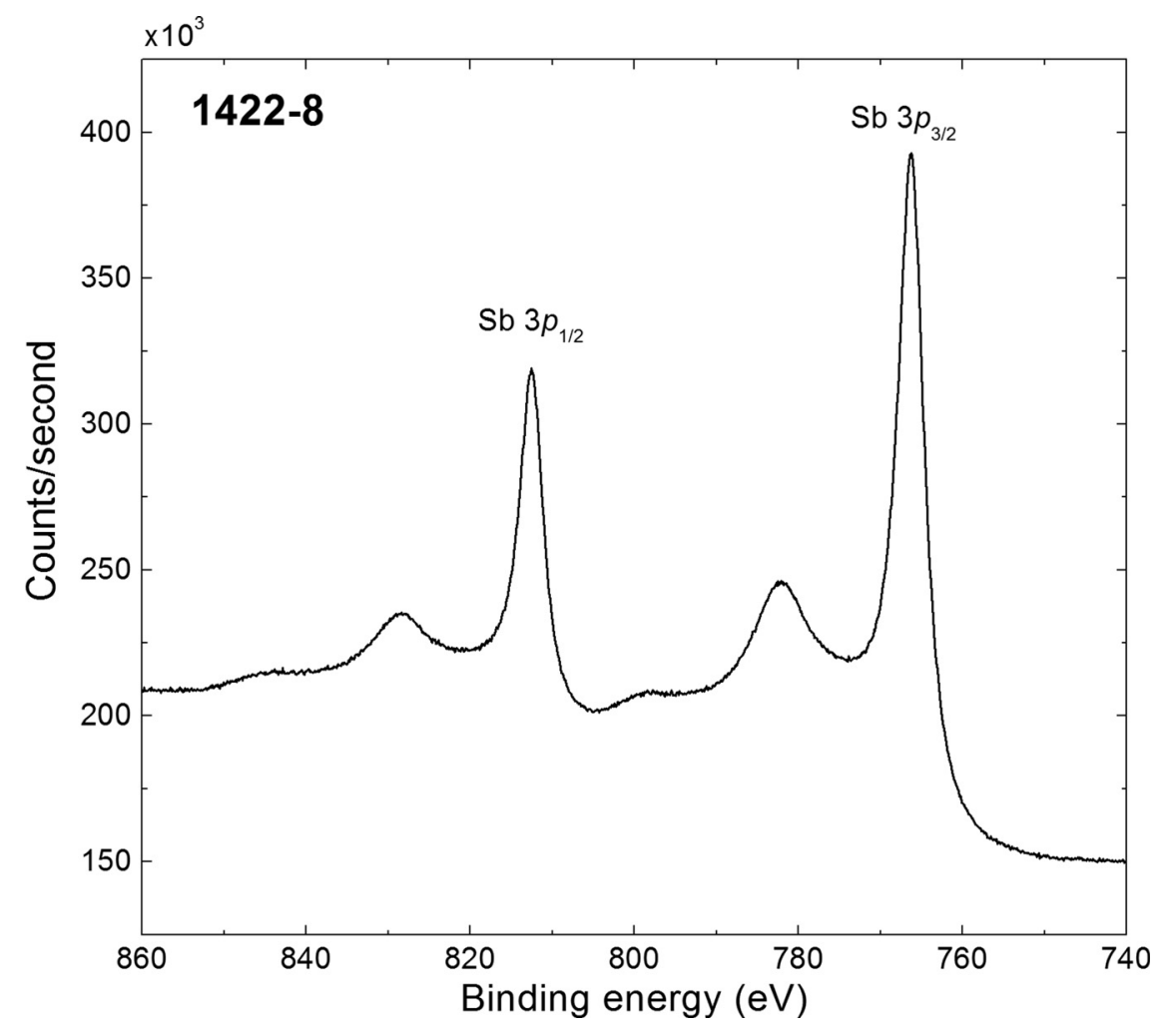

Accession \#: 1422-08

- Host Material: Antimony (Sb)

- Technique: XPS

- Spectral Region: Sb 3p

Instrument: Thermo Scientific K-Alpha+

Excitation Source: Al Ka monochromatic

Source Energy: $1486.6 \mathrm{eV}$

Source Strength: $72 \mathrm{~W}$

Source Size: $0.004 \times 0.004 \mathrm{~mm}$

Analyzer Type: Double focussing hemispherical analyzer

Incident Angle: $60^{\circ}$

Emission Angle: $90^{\circ}$

Analyzer Pass Energy: $40 \mathrm{eV}$

Analyzer Resolution: $0.1 \mathrm{eV}$

Total Signal Accumulation Time: $120 \mathrm{~s}$

Total Elapsed Time: $153 \mathrm{~s}$

Number of Scans: 20

Effective Detector Width: $0.1 \mathrm{eV}$

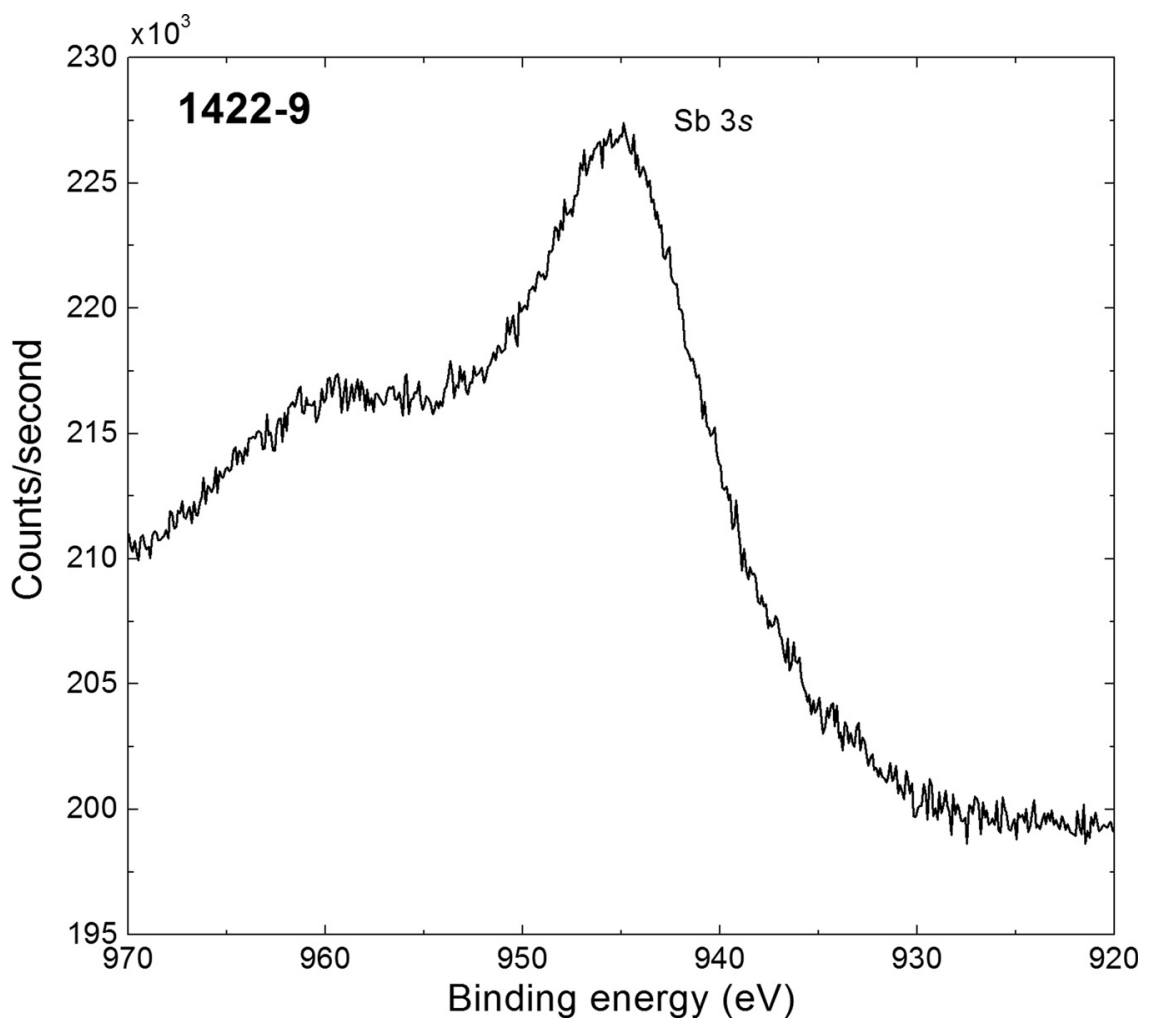

Accession \#: 1422-09

Host Material: Antimony (Sb)

Technique: XPS

- Spectral Region: Sb 3s

Instrument: Thermo Scientific K-Alpha+

Excitation Source: Al Ka monochromatic

Source Energy: $1486.6 \mathrm{eV}$

Source Strength: $72 \mathrm{~W}$

Source Size: $0.004 \times 0.004 \mathrm{~mm}$

Analyzer Type: Double focussing hemispherical analyzer

Incident Angle: $60^{\circ}$

Emission Angle: $90^{\circ}$

Analyzer Pass Energy: $40 \mathrm{eV}$

Analyzer Resolution: $0.1 \mathrm{eV}$

Total Signal Accumulation Time: $120 \mathrm{~s}$

Total Elapsed Time: $153 \mathrm{~s}$

Number of Scans: 20

Effective Detector Width: $0.1 \mathrm{eV}$ 


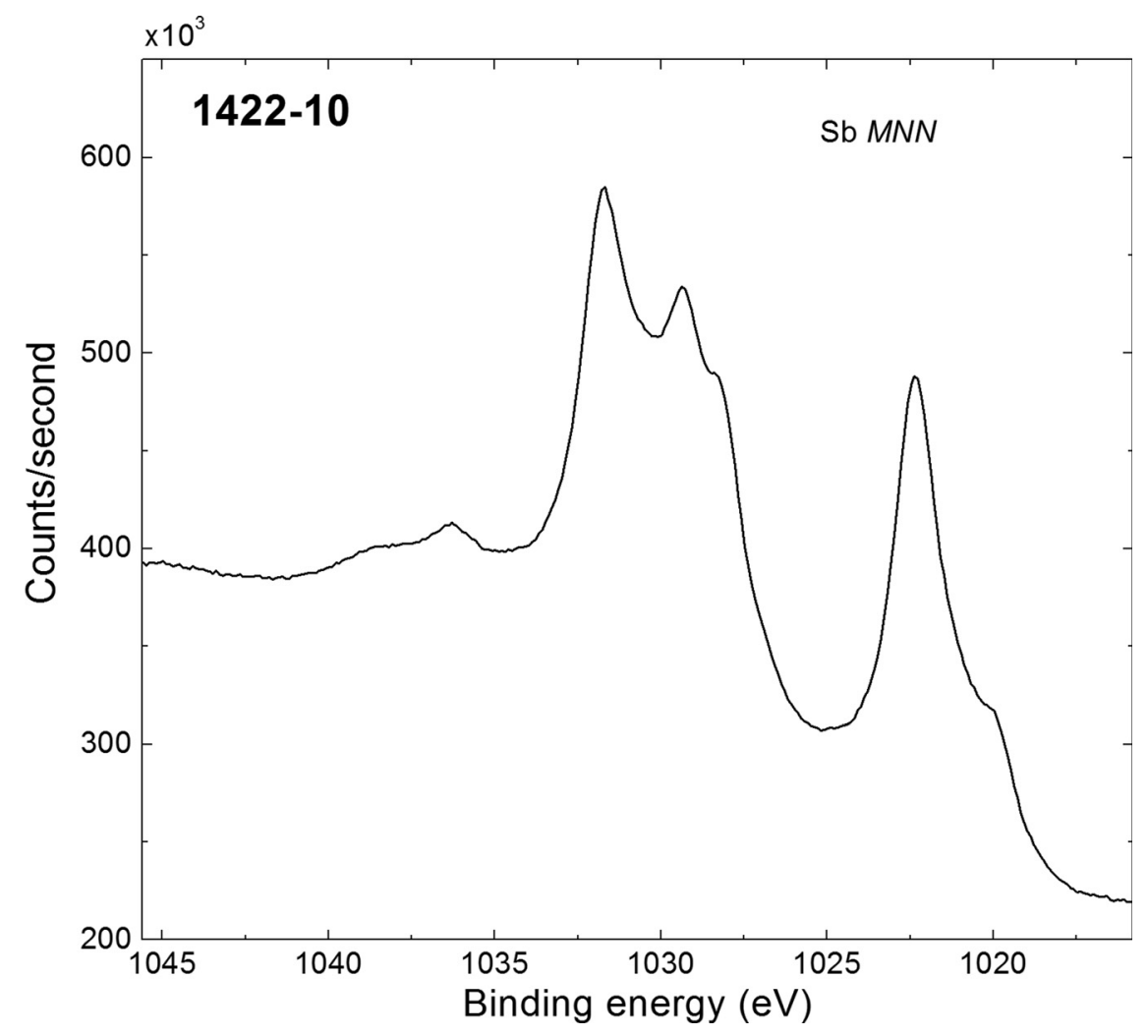

- Accession \#: 1422-10

- Host Material: Antimony (Sb)

- Technique: XPS

- Spectral Region: Sb MNN

Instrument: Thermo Scientific K-Alpha+

Excitation Source: Al Ka monochromatic

Source Energy: $1486.6 \mathrm{eV}$

Source Strength: $72 \mathrm{~W}$

Source Size: $0.004 \times 0.004 \mathrm{~mm}$

Analyzer Type: Double focussing hemispherical analyzer

Incident Angle: $60^{\circ}$

Emission Angle: $90^{\circ}$

Analyzer Pass Energy: $40 \mathrm{eV}$

Analyzer Resolution: $0.1 \mathrm{eV}$

Total Signal Accumulation Time: $120 \mathrm{~s}$

Total Elapsed Time: $153 \mathrm{~s}$

Number of Scans: 20

Effective Detector Width: $0.1 \mathrm{eV}$ 